\title{
On addition and multiplication of points in a certain class of projective Klingenberg planes
}

\author{
Basri Çelik and Fatma Özen Erdoğan
}

"Correspondence:

basri@uludag.edu.tr

Department of Mathematics,

Faculty of Art and Science, Uludağ

University, Bursa, Görükle, Turkey

\begin{abstract}
Let $(O, E, U, V)$ be the coordination quadruple of the projective Klingenberg plane (PK-plane) coordinated with dual quaternion ring $Q(\varepsilon)=Q+Q \varepsilon=\{x+y \varepsilon \mid x, y \in Q\}$, where $Q$ is any quaternion ring over a field. In this paper, we define addition and multiplication of points on the line $O U=[0,1,0]$ geometrically, also we give the algebraic correspondences of them. Finally, we carry over some well-known properties of ordinary addition and multiplication to our definition.
\end{abstract}

MSC: $51 \mathrm{CO} 5 ; 51 \mathrm{~N} 35 ; 14 \mathrm{~A} 22 ; 16 \mathrm{~L} 30$

Keywords: projective Klingenberg plane; local ring; geometric addition; geometric multiplication

\section{Introduction}

Algebra and geometry are two essential subjects of mathematics and therefore relations between these subjects have been investigated since Euclides of Alexandra B.C. 325. In this paper, our aim is to construct a relation between algebraic and geometric definitions of addition and multiplication of points in projective Klingenberg planes which seem to be a generalization of ordinary projective planes. In this section, we give some required concepts from the literature.

A ring $\mathbf{R}$ with an identity element is called local if the set $\mathbf{I}$ of its non-units is an ideal.

A projective plane (see [1]) $(\mathcal{P}, \mathcal{L}, \in)$ is a system in which the elements of $\mathcal{P}$ are called points and the elements of $\mathcal{L}$ are called lines together with an incidence relation $\epsilon$ between the points and lines such that

P1: If $P \neq Q$ and $P, Q \in \mathcal{P}$, then there is a unique line passing through $P$ and $Q$ (denoted by $P \vee Q$ or $P Q)$.

P2: If $l, m \in \mathcal{L}$, then there exists at least one point on both $l$ and $m$.

P3: There exist four points such that no three of them are collinear.

It is proven that there exists a unique intersection point of different lines.

A projective Klingenberg plane (PK-plane) (see $[2,3])$ is a system $(\mathcal{P}, \mathcal{L}, \epsilon, \sim)$ where $(\mathcal{P}, \mathcal{L}, \epsilon)$ is an incidence structure and $\sim$ is an equivalence relation on $\mathcal{P} \cup \mathcal{L}$ (called neighboring) such that no point is neighbor to any line and the following axioms are satisfied:

PK1: If $P \nsim Q, P, Q \in \mathcal{P}$, then there is a unique line passing through $P$ and $Q$ (denoted by $P \vee Q$ or $P Q)$.

PK2: If $l \nsim m, l, m \in \mathcal{L}$, then there is a unique point on both $l$ and $m$ (denoted by $l \wedge m$ or $\operatorname{lm})$.

(0) 2013 Celik and Erdoğan; licensee Springer. This is an Open Access article distributed under the terms of the Creative Commons Attribution License (http://creativecommons.org/licenses/by/2.0), which permits unrestricted use, distribution, and reproduction in any medium, provided the original work is properly cited. 
PK3: There is a projective plane $\Pi^{*}$ and an incidence structure epimorphism $\chi: \Pi \rightarrow$ $\Pi^{*}$ such that $P \sim Q \Leftrightarrow \chi(P)=\chi(Q)$ and $l \sim m \Leftrightarrow \chi(l)=\chi(m)$.

A point $P \in \mathcal{P}$ is called near a line $g \in \mathcal{L}$ (which is denoted by $P \sim g$ ) iff there exists a line $h \sim g$ such that $P \in h$.

Now we give two theorems and a corollary from [2].

Theorem 1 Let $\Pi$ be a PK-plane with a canonical image $\Pi^{*}$. Choose a basis $(O, U, V, E)$ consisting of four points whose image $(\chi(O), \chi(U), \chi(V), \chi(E))$ in $\Pi^{*}$ forms a quadrangle. Let $g_{\infty}:=U V, l:=O E, W:=l \wedge U V, \eta:=\{P \in l \mid P \sim O\}$ and $R:=\{P \in l \mid P \nsim W\}$. Let $0:=O, 1:=E$. Then the points $P \in \mathcal{P}$ and the lines $g \in \mathcal{L}$ of $\Pi$ get their coordinates as follows:

$$
\begin{aligned}
& \text { If } P \nsim g_{\infty}, \text { let } P=(x, y, 1) \text { where }(x, x, 1)=P V \wedge l,(y, y, 1)=P U \wedge l . \\
& \text { If } P \sim g_{\infty}, P \nsim V \text {, let } P=(1, y, z) \text { where }(1, z, 1)=((P V \wedge U E) \vee O) \wedge E V \text { and } \\
& (1, y, 1)=O P \wedge E V . \\
& \text { If } P \sim V \text {, let } P=(w, 1, z) \text { where }(1,1, z)=P U \wedge l \text {, and }(w, 1,1)=O P \wedge E U(\text { clearly, } \\
& \quad w, z \in \eta) \text {. } \\
& \text { If } g \nsim V \text {, then } g=[m, 1, p] \text { where }(1, m, 1)=\left(\left(g \wedge g_{\infty}\right) \vee O\right) \wedge E V,(0, p, 1)=g \wedge O V . \\
& \text { If } g \sim V, g \nsim g_{\infty}, \text { then } g=[1, u, v] \text { where }(u, 1,1)=\left(\left(g \wedge g_{\infty}\right) \vee O\right) \wedge E U, \\
& \quad(v, 0,1)=g \wedge O U \text {. } \\
& \text { If } g \sim g_{\infty}, \text { then } g=[q, r, 1] \text { where }(1,0, q)=g \wedge O U,(0,1, r)=g \wedge O V .(\text { Then } q, r \in \eta .) \\
& \text { Then } O=(0,0,1), U=(1,0,0), V=(0,1,0), E=(1,1,1), \text { OU }=[0,1,0], \text { OV }=[1,0,0], \\
& U V=[0,0,1], l=O E=[1,1,0] \text { and } a \text { point } a \in R \text { has coordinates }(a, a, 1) \text {. We note that } \\
& \left(a_{1}, a_{2}, a_{3}\right) \sim\left(b_{1}, b_{2}, b_{3}\right) \text { if and only if } a_{i}-b_{i} \in \mathbf{I}, \text { for } i=1,2,3, \text { dually for lines. }
\end{aligned}
$$

Theorem 2 Let $\mathbf{R}$ be a local ring, and the set of the non-units is denoted by $\mathbf{I}$. The system $(\mathcal{P}, \mathcal{L}, \in, \sim)$ is a PK-plane where

$$
\begin{aligned}
& \mathcal{P}=\{(x, y, 1) \mid x, y \in \mathbf{R}\} \cup\{(1, y, z) \mid y \in \mathbf{R}, z \in \mathbf{I}\} \cup\{(w, 1, z) \mid w, z \in \mathbf{I}\}, \\
& \mathcal{L}=\{[m, 1, k] \mid m, k \in \mathbf{R}\} \cup\{[1, n, p] \mid n \in \mathbf{I}, p \in \mathbf{R}\} \cup\{[q, n, 1] \mid q, n \in \mathbf{I}\}, \\
& (x, y, 1) \in[m, 1, k] \Leftrightarrow y=x m+k, \\
& (x, y, 1) \in[1, n, p] \Leftrightarrow x=y n+p, \\
& (x, y, 1) \notin[q, n, 1], \\
& (1, y, z) \in[m, 1, k] \Leftrightarrow y=m+z k \\
& (1, y, z) \in[q, n, 1] \quad \Leftrightarrow \quad z=q+y n, \\
& (1, y, z) \notin[1, n, p], \\
& (w, 1, z) \in[1, n, p] \Leftrightarrow w=n+z p, \\
& (w, 1, z) \in[q, n, 1] \quad \Leftrightarrow \quad z=w q+n, \\
& (w, 1, z) \notin[m, 1, k], \\
& \left(x_{1}, x_{2}, x_{3}\right) \sim\left(y_{1}, y_{2}, y_{3}\right) \Leftrightarrow x_{i}-y_{i} \in \mathbf{I}, \\
& {\left[a_{1}, a_{2}, a_{3}\right] \sim\left[b_{1}, b_{2}, b_{3}\right] \quad \Leftrightarrow \quad a_{i}-b_{i} \in \mathbf{I} .}
\end{aligned}
$$


Corollary 3 If $t \in \mathbf{I}$, then $1-t$ is a unit. Therefore

$$
\begin{aligned}
& (x, y, 1) \nsim(1, y, z), \quad(x, y, 1) \nsim(w, 1, z), \quad(1, y, z) \nsim(w, 1, z), \\
& (w, 1, z) \sim(u, 1, t), \quad(x, y, 1) \sim(u, v, 1) \quad \Leftrightarrow \quad x-u \in \mathbf{I}, \quad y-v \in \mathbf{I}, \\
& (1, y, z) \sim(1, u, t) \quad \Leftrightarrow \quad y-u \in \mathbf{I} .
\end{aligned}
$$

The PK-plane which is given in Theorem 1 is denoted with $P K_{2}(\mathbf{R})$ and it is called the PK-plane coordinated with (the local ring) $\mathbf{R}$.

Finally, we recall some definitions and theorems from [4]. Let $\mathbf{Q}=\left\{x_{0}+x_{1} i+x_{2} j+x_{3} k \mid\right.$ $\left.x_{0}, x_{1}, x_{2}, x_{3} \in \mathbf{F}\right\}$ be an arbitrary division ring over a field $\mathbf{F}$ (which is called the ring of quaternions over $\mathbf{F}$ ) with the operators addition and multiplication defined by

$$
\begin{aligned}
\left(x_{0}\right. & \left.+x_{1} i+x_{2} j+x_{3} k\right)+\left(y_{0}+y_{1} i+y_{2} j+y_{3} k\right) \\
= & \left(x_{0}+y_{0}\right)+\left(x_{1}+y_{1}\right) i+\left(x_{2}+y_{2}\right) j+\left(x_{3}+y_{3}\right) k, \\
\left(x_{0}+\right. & \left.x_{1} i+x_{2} j+x_{3} k\right) \cdot\left(y_{0}+y_{1} i+y_{2} j+y_{3} k\right) \\
= & \left(x_{0} y_{0}-x_{1} y_{1}-x_{2} y_{2}-x_{3} y_{3}\right)+\left(x_{0} y_{1}+x_{1} y_{0}+x_{2} y_{3}-x_{3} y_{2}\right) i \\
\quad & \quad\left(x_{0} y_{2}+x_{2} y_{0}+x_{3} y_{1}-x_{1} y_{3}\right) j+\left(x_{0} y_{3}+x_{3} y_{0}+x_{1} y_{2}-x_{2} y_{1}\right) k .
\end{aligned}
$$

For detailed information about quaternions, see [5].

We consider the set $\mathbf{Q}(\varepsilon)=\mathbf{Q}+\mathbf{Q} \varepsilon=\{a+b \varepsilon \mid a, b \in \mathbf{Q}\}$ together with the operations

$$
\begin{aligned}
& (a+b \varepsilon)+(c+d \varepsilon)=(a+c)+(b+d) \varepsilon, \\
& (a+b \varepsilon)(c+d \varepsilon)=a c+(a d+b c) \varepsilon .
\end{aligned}
$$

Then the elements of $\mathbf{Q}(\varepsilon)$ are called dual quaternions.

Theorem 4 The non-unit elements of $\mathbf{Q}(\varepsilon)$ are in the form $b \varepsilon$, for $b \in \mathbf{Q}$ and if $a \neq 0$, $a, b \in \mathbf{Q}, a+b \varepsilon$ is a unit and $(a+b \varepsilon)^{-1}=a^{-1}-a^{-1} b a^{-1} \varepsilon$.

Theorem 5 The set of non-units $\mathbf{I}=\mathbf{Q} \varepsilon=\{b \varepsilon \mid b \in \mathbf{Q}\}$ is an ideal of $\mathbf{Q}(\varepsilon)$.

Corollary 6 The following properties are valid:

(i) $\mathbf{Q}(\varepsilon)$ is a local ring (and it is called the dual local ring on $\mathbf{Q}$ ).

(ii) From Theorem $2, P K_{2}(\mathbf{Q}(\varepsilon))=(\mathcal{P}, \mathcal{L}, \epsilon, \sim)$ is a PK-plane, where

$$
\begin{aligned}
\mathcal{P}= & \left\{\left(x_{1}+x_{2} \varepsilon, y_{1}+y_{2} \varepsilon, 1\right) \mid x_{1}, x_{2}, y_{1}, y_{2} \in \mathbf{Q}\right\} \\
& \cup\left\{\left(1, y_{1}+y_{2} \varepsilon, z_{2} \varepsilon\right) \mid y_{1}, y_{2}, z_{2} \in \mathbf{Q}\right\} \cup\left\{\left(w_{2} \varepsilon, 1, z_{2} \varepsilon\right) \mid w_{2}, z_{2} \in \mathbf{Q}\right\}
\end{aligned}
$$

and

$$
\begin{aligned}
\mathcal{L}=\{ & {\left.\left[m_{1}+m_{2} \varepsilon, 1, k_{1}+k_{2} \varepsilon\right] \mid m_{1}, m_{2}, k_{1}, k_{2} \in \mathbf{Q}\right\} } \\
& \cup\left\{\left[1, n_{2} \varepsilon, p_{1}+p_{2} \varepsilon\right] \mid n_{2}, p_{1}, p_{2} \in \mathbf{Q}\right\} \cup\left\{\left[q_{2} \varepsilon, n_{2} \varepsilon, 1\right] \mid q_{2}, n_{2} \in \mathbf{Q}\right\} .
\end{aligned}
$$

Theorem 7 Neighbor relation $\sim$ is an equivalence relation over $\mathcal{P}$ and $\mathcal{L}$ in $P K_{2}(\mathbf{Q}(\varepsilon))$. 
Theorem 8 In $P K_{2}(\mathbf{Q}(\varepsilon))$, the following properties are satisfied:

(i) $\left(x_{1}+x_{2} \varepsilon, y_{1}+y_{2} \varepsilon, 1\right) \in\left[m_{1}+m_{2} \varepsilon, 1, k_{1}+k_{2} \varepsilon\right] \Leftrightarrow y_{1}=x_{1} m_{1}+k_{1}$, $y_{2}=x_{2} m_{1}+x_{1} m_{2}+k_{2}$.

(ii) $\left(x_{1}+x_{2} \varepsilon, y_{1}+y_{2} \varepsilon, 1\right) \in\left[1, n_{2} \varepsilon, p_{1}+p_{2} \varepsilon\right] \Leftrightarrow x_{1}=p_{1}, x_{2}=y_{1} n_{2}+p_{2}$.

(iii) $\left(1, y_{1}+y_{2} \varepsilon, z_{2} \varepsilon\right) \in\left[m_{1}+m_{2} \varepsilon, 1, k_{1}+k_{2} \varepsilon\right] \Leftrightarrow y_{1}=m_{1}, y_{2}=m_{2}+z_{2} k_{1}$.

(iv) $\left(1, y_{1}+y_{2} \varepsilon, z_{2} \varepsilon\right) \in\left[q_{2} \varepsilon, n_{2} \varepsilon, 1\right] \Leftrightarrow z_{2}=q_{2}+y_{1} n_{2}$.

(v) $\left(w_{2} \varepsilon, 1, z_{2} \varepsilon\right) \in\left[1, n_{2} \varepsilon, p_{1}+p_{2} \varepsilon\right] \Leftrightarrow w_{2}=n_{2}+z_{2} p_{1}$.

(vi) $\left(w_{2} \varepsilon, 1, z_{2} \varepsilon\right) \in\left[q_{2} \varepsilon, n_{2} \varepsilon, 1\right] \Leftrightarrow z_{2}=n_{2}$.

(vii) $\left(a_{1}+a_{2} \varepsilon, b_{1}+b_{2} \varepsilon, 1\right) \sim\left(c_{1}+c_{2} \varepsilon, d_{1}+d_{2} \varepsilon, 1\right) \Leftrightarrow c_{1}=a_{1} \wedge d_{1}=b_{1}$.

(viii) $\left(1, a_{1}+a_{2} \varepsilon, b_{2} \varepsilon\right) \sim\left(1, c_{1}+c_{2} \varepsilon, d_{2} \varepsilon\right) \Leftrightarrow c_{1}=a_{1}$.

(ix) For every $a_{2}, b_{2}, c_{2}, d_{2} \in \mathbf{Q}\left(a_{2} \varepsilon, 1, b_{2} \varepsilon\right) \sim\left(c_{2} \varepsilon, 1, d_{2} \varepsilon\right)$.

\section{Addition and multiplication of points on the line $\mathrm{OU}$ in $P K_{2}(\mathrm{Q}(\varepsilon)$ )}

In this section we give the definition of addition and multiplication of points on the line $O U$, and also we give some useful results for calculating addition and multiplication of points where $(O, U, V, E)$ is a base of $P K_{2}(\mathbf{Q}(\varepsilon))$.

Definition 9 Let $A$ and $B$ be non-neighbor points of $P K_{2}(\mathbf{Q}(\varepsilon))$ on the line $O U$. Then

(i) $A+B$ is defined as the intersection point of the lines $L V$ and $O U$ where $L=K U \wedge B S, K=A V \wedge O S, S=(1,1,0)$.

(ii) $A \cdot B$ is defined as the intersection point of the lines $V N$ and $O U$ where $N=A S \wedge O M, M=B V \wedge 1 S, S=(1,1,0), 1=(1,0,1)$.

Now we state a theorem which interprets Definition 9 algebraically.

Theorem 10 Let $A=\left(a_{1}+a_{2} \varepsilon, 0,1\right)$ and $B=\left(b_{1}+b_{2} \varepsilon, 0,1\right)$ be two non-neighbor points on the line $O U$ and let $Z=\left(1,0, z_{2} \varepsilon\right)$ be the point on the line $O U$ (neighbor to $U$ ). Then:

(i) $A+B=\left(\left(a_{1}+b_{1}\right)+\left(a_{2}+b_{2}\right) \varepsilon, 0,1\right)$.

(ii) $A+Z=\left(1,0, z_{2} \varepsilon\right)$.

(iii) $A \cdot B=\left(a_{1} b_{1}+\left(a_{1} b_{2}+a_{2} b_{1}\right) \varepsilon, 0,1\right)$.

(iv) $A \cdot Z=\left(1,0,\left(z_{2} a_{1}^{-1}\right) \varepsilon\right)$ where $A \nsim O$.

(v) $Z \cdot A=\left(1,0,\left(a_{1}^{-1} z_{2}\right) \varepsilon\right)$ where $A \nsim O$.

Proof Proof can be done following Definition 9 by using simple calculations.

(i)

$$
\begin{aligned}
A+B & =((A V \wedge O S) U \wedge B S) V \wedge O U \\
& =\left[1,0,\left(a_{1}+b_{1}\right)+\left(a_{2}+b_{2}\right) \varepsilon\right] \wedge[0,1,0] \\
& =\left(\left(a_{1}+b_{1}\right)+\left(a_{2}+b_{2}\right) \varepsilon, 0,1\right) .
\end{aligned}
$$

(ii)

$$
\begin{aligned}
A+Z & =((A V \wedge O S) U \wedge Z S) V \wedge O U \\
& =\left[z_{2} \varepsilon, 0,1\right] \wedge[0,1,0] \\
& =\left(1,0, z_{2} \varepsilon\right)=Z .
\end{aligned}
$$


(iii) If $B \nsim O$, then the inverse of $b_{1}$ exists and therefore

$$
\begin{aligned}
A \cdot B & =((B V \wedge 1 S) O \wedge A S) V \wedge O U \\
& =\left[1,0, a_{1} b_{1}+\left(\left(\left(a_{1} b_{1}\right)\left(b_{1}^{-1} b_{2} b_{1}^{-1}\right)\right) b_{1}+a_{2} b_{1}\right) \varepsilon\right] \wedge[0,1,0] \\
& =\left(a_{1} b_{1}+\left(\left(\left(a_{1} b_{1}\right)\left(b_{1}^{-1} b_{2} b_{1}^{-1}\right)\right) b_{1}+a_{2} b_{1}\right) \varepsilon, 0,1\right)
\end{aligned}
$$

is obtained. Then using the associative and inversive property in $\mathbf{Q}$, we find $A \cdot B=\left(a_{1} b_{1}+\right.$ $\left.\left(a_{1} b_{2}+a_{2} b_{1}\right) \varepsilon, 0,1\right)$.

If $B \sim O$, then

$$
\begin{aligned}
A \cdot B & =((B V \wedge 1 S) O \wedge A S) V \wedge O U \\
& =\left[1,0, a_{1} b_{2} \varepsilon,\right] \wedge[0,1,0] \\
& =\left(a_{1} b_{2} \varepsilon, 0,1\right) .
\end{aligned}
$$

(iv)

$$
\begin{aligned}
A \cdot Z & =((Z V \wedge 1 S) O \wedge A S) V \wedge O U \\
& =\left[\left(z_{2} \varepsilon\right)\left(a_{1}+a_{2} \varepsilon\right)^{-1}, 0,1\right] \wedge[0,1,0] \\
& =\left(1,0,\left(z_{2} \varepsilon\right)\left(a_{1}+a_{2} \varepsilon\right)^{-1}\right) \\
& =\left(1,0,\left(z_{2} a_{1}^{-1}\right) \varepsilon\right)
\end{aligned}
$$

is obtained.

(v)

$$
\begin{aligned}
Z \cdot A & =((A V \wedge 1 S) O \wedge Z S) V \wedge O U \\
& =\left[\left(a_{1}^{-1} z_{2}\right) \varepsilon, 0,1\right] \wedge[0,1,0] \\
& =\left(1,0,\left(a_{1}^{-1} z_{2}\right) \varepsilon\right)
\end{aligned}
$$

is obtained.

Theorem 11 The properties given in Theorem 10 are independent of the choice of the point $S$ given in Definition 9, where $S$ is a point on $U V$ and $S \nsim V, S \nsim U$.

Proof If $S^{\prime}$ is an arbitrary point on $U V$ non-neighbor to $V$, then there exist $s_{1}, s_{2} \in \mathbf{Q}$ such that $S^{\prime}=\left(1, s_{1}+s_{2} \varepsilon, 0\right)$. We must show that the properties given in Theorem 10 hold when we replace $S$ by $S^{\prime}$.

(i)

$$
\begin{aligned}
A+B & =\left(\left(A V \wedge O S^{\prime}\right) U \wedge B S^{\prime}\right) V \wedge O U \\
& =\left[1,0,\left(a_{1}+b_{1}\right)+\left(a_{2}+b_{2}\right) \varepsilon\right] \wedge[0,1,0] \\
& =\left(\left(a_{1}+b_{1}\right)+\left(a_{2}+b_{2}\right) \varepsilon, 0,1\right)
\end{aligned}
$$

is obtained. 
(ii)

$$
\begin{aligned}
A+Z & =\left(\left(A V \wedge O S^{\prime}\right) U \wedge Z S^{\prime}\right) V \wedge O U \\
& =\left[z_{2} \varepsilon, 0,1\right] \wedge[0,1,0] \\
& =\left(1,0, z_{2} \varepsilon\right)=Z
\end{aligned}
$$

is obtained.

(iii) If $B \nsim O$, then $b_{1}^{-1}$ exists and therefore

$$
\begin{aligned}
A \cdot B & =\left(\left(B V \wedge 1 S^{\prime}\right) O \wedge A S^{\prime}\right) V \wedge O U \\
& =\left[1,0, a_{1} b_{1}+\left(\left(\left(a_{1} b_{1}\right)\left(b_{1}^{-1} b_{2} b_{1}^{-1}\right)\right) b_{1}+a_{2} b_{1}\right) \varepsilon\right] \wedge[0,1,0] \\
& =\left(a_{1} b_{1}+\left(\left(\left(a_{1} b_{1}\right)\left(b_{1}^{-1} b_{2} b_{1}^{-1}\right)\right) b_{1}+a_{2} b_{1}\right) \varepsilon, 0,1\right)
\end{aligned}
$$

is obtained. Then using the associative and inversive property in $\mathbf{Q}$, we find $A \cdot B=\left(a_{1} b_{1}+\right.$ $\left.\left(a_{1} b_{2}+a_{2} b_{1}\right) \varepsilon, 0,1\right)$.

If $B \sim O$, then

$$
A \cdot B=\left(\left(B V \wedge 1 S^{\prime}\right) O \wedge A S^{\prime}\right) V \wedge O U=\left[1,0, a_{1} b_{2} \varepsilon,\right] \wedge[0,1,0]=\left(a_{1} b_{2} \varepsilon, 0,1\right)
$$

(iv)

$$
\begin{aligned}
A \cdot Z & =\left(\left(Z V \wedge 1 S^{\prime}\right) O \wedge A S^{\prime}\right) V \wedge O U \\
& =\left[\left(z_{2} \varepsilon\right)\left(a_{1}+a_{2} \varepsilon\right)^{-1}, 0,1\right] \wedge[0,1,0] \\
& =\left(1,0,\left(z_{2} \varepsilon\right)\left(a_{1}+a_{2} \varepsilon\right)^{-1}\right. \\
& =\left(1,0,\left(z_{2} a_{1}^{-1}\right) \varepsilon\right) .
\end{aligned}
$$

(v)

$$
\begin{aligned}
Z \cdot A & =\left(\left(A V \wedge 1 S^{\prime}\right) O \wedge Z S^{\prime}\right) V \wedge O U \\
& =\left[\left(a_{1}^{-1} z_{2}\right) \varepsilon, 0,1\right] \wedge[0,1,0]=\left(1,0,\left(a_{1}^{-1} z_{2}\right) \varepsilon\right) .
\end{aligned}
$$

Theorem 12 Let $A$ and $B$ be two non-neighbor points on $O U$ and $A^{*} \sim A, B^{*} \sim B$, then $A+B \sim A^{*}+B^{*}, A \cdot B \sim A^{*} \cdot B^{*}$.

Proof Let $A=\left(a_{1}+a_{2} \varepsilon, 0,1\right), A^{*}=\left(a_{1}^{*}+a_{2}^{*} \varepsilon, 0,1\right), B=\left(b_{1}+b_{2} \varepsilon, 0,1\right)$ and $B^{*}=\left(b_{1}^{*}+b_{2}^{*} \varepsilon, 0,1\right)$. We obtain $A+B=\left(\left(a_{1}+b_{1}\right)+\left(a_{2}+b_{2}\right) \varepsilon, 0,1\right)$ and $A^{*}+B^{*}=\left(\left(a_{1}^{*}+b_{1}^{*}\right)+\left(a_{2}^{*}+b_{2}^{*}\right) \varepsilon, 0,1\right)$. Then we have

$$
\begin{aligned}
A^{*} \sim A, \quad B^{*} \sim B & \Leftrightarrow a_{1}^{*}=a_{1}, \quad b_{1}^{*}=b_{1} \\
& \Leftrightarrow a_{1}^{*}+b_{1}^{*}=a_{1}+b_{1} \\
& \Leftrightarrow A^{*}+B^{*} \sim A+B .
\end{aligned}
$$


Since $A \cdot B=\left(a_{1} b_{1}+\left(a_{1} b_{2}+a_{2} b_{1}\right) \varepsilon, 0,1\right)$ and $A^{*} \cdot B^{*}=\left(a_{1}^{*} b_{1}^{*}+\left(a_{1}^{*} b_{2}^{*}+a_{2}^{*} b_{1}^{*}\right) \varepsilon, 0,1\right)$, we get

$$
\begin{aligned}
A^{*} \sim A, \quad B^{*} \sim B & \Leftrightarrow a_{1}^{*}=a_{1}, \quad b_{1}^{*}=b_{1} \\
& \Leftrightarrow a_{1}^{*} \cdot b_{1}^{*}=a_{1} \cdot b_{1} \\
& \Leftrightarrow A^{*} \cdot B^{*} \sim A \cdot B .
\end{aligned}
$$

If $Z=\left(1,0, z_{2} \varepsilon\right)$ and $Z^{*}=\left(1,0, z_{2}^{*} \varepsilon\right)$, then $A+Z=Z$ and $A^{*}+Z^{*}=Z^{*}$ and it is trivial that

$$
Z \sim Z^{*} \quad \Leftrightarrow \quad A+Z \sim A^{*}+Z^{*}
$$

Similarly, we have

$$
A \cdot Z=\left(1,0,\left(z_{2} a_{1}^{-1}\right) \varepsilon\right) \sim\left(1,0,\left(z_{2}^{*} a_{1}^{*}-1\right) \varepsilon\right)=A^{*} \cdot Z^{*}
$$

and

$$
Z \cdot A=\left(1,0,\left(a_{1}^{-1} z_{2}\right) \varepsilon\right) \sim\left(1,0,\left(a_{1}^{*}-1 z_{2}^{*}\right) \varepsilon\right)=Z^{*} \cdot A^{*}
$$

Corollary 13 The following statements are valid where the points $A, B, Z, O$ are defined as in Theorem 10 and $Y$ is a point neighbor to $(0,0,1)$ (i.e., $\left.Y \in\left\{\left(y_{2} \varepsilon, 0,1\right) \mid y_{2} \in \mathbf{Q}\right\}\right)$ :

(i) $A+B=B+A$ and $A+Z=Z+A$.

(ii) $A+O=A$ and $O+Z=Z$.

(iii) $A+Y \sim A$.

(iv) $A \cdot B \neq B \cdot A$.

(v) $O \cdot A=A \cdot O=O$.

(vi) $1 \cdot A=A=A \cdot 1$ and $1 \cdot Z=Z=Z \cdot 1$.

(vii) $A \cdot Y \sim Y$ and $Y \cdot A \sim Y$.

Proof (i) Since

$$
\begin{aligned}
((A V \wedge O S) U \wedge B S) V & =\left[1,0,\left(a_{1}+b_{1}\right)+\left(a_{2}+b_{2}\right) \varepsilon\right] \\
& =\left[1,0,\left(b_{1}+a_{1}\right)+\left(b_{2}+a_{2}\right) \varepsilon\right] \\
& =((B V \wedge O S) U \wedge A S) V
\end{aligned}
$$

we obtain $A+B=B+A$. And since

$$
((A V \wedge O S) U \wedge Z S) V=\left[z_{2} \varepsilon, 0,1\right]=((Z V \wedge O S) U \wedge A S) V
$$

we obtain $A+Z=Z+A$.

(ii)

$$
A+O=((A V \vee O S) U \wedge O S) V \wedge O U=\left[1,0, a_{1}+a_{2} \varepsilon\right] \wedge[0,1,0]=A
$$

is obtained. 
Similarly, we get

$$
\begin{aligned}
O+Z & =((O V \wedge O S) U \wedge Z S) V \wedge O U \\
& =\left[z_{2} \varepsilon, 0,1\right] \wedge[0,1,0]=Z
\end{aligned}
$$

(iii) It is trivial from Theorem 12.

(iv) Let $A=(i, 0,1)$ and $B=(j, 0,1)$. Since $A \cdot B=(k, 0,1)$ and $B \cdot A=(-k, 0,1)$, we have $A \cdot B \neq B \cdot A$.

(v) By simple calculations,

$$
\begin{aligned}
O \cdot A & =((A V \wedge 1 S) O \wedge O S) V \wedge O U \\
& =[1,0,0] \wedge[0,1,0] \\
& =((O V \wedge 1 S) O \wedge A S) V \wedge O U \\
& =A \cdot O
\end{aligned}
$$

is obtained.

Also, since $[1,0,0] \wedge[0,1,0]=(0,0,1)=O$, we have $O \cdot A=A \cdot O=O$.

(vi) Since

$$
\begin{aligned}
A \cdot 1 & =((1 V \wedge 1 S) O \wedge A S) V \wedge O U \\
& =\left[1,0, a_{1}+a_{2} \varepsilon\right] \wedge[0,1,0] \\
& =((A V \wedge 1 S) O \wedge 1 S) V \wedge O U \\
& =1 \cdot A
\end{aligned}
$$

and $\left[1,0, a_{1}+a_{2} \varepsilon\right] \wedge[0,1,0]=\left(a_{1}+a_{2} \varepsilon, 0,1\right)=A$, we get $A \cdot 1=1 \cdot A=A$.

Similarly,

$$
\begin{aligned}
1 \cdot Z & =((Z V \wedge 1 S) O \wedge 1 S) V \wedge O U \\
& =\left[z_{2} \varepsilon, 0,1\right] \wedge[0,1,0]=Z \cdot 1
\end{aligned}
$$

and hence we get $\left[z_{2} \varepsilon, 0,1\right] \wedge[0,1,0]=\left(1,0, z_{2} \varepsilon\right)=Z$.

(vii) Since $Y=\left(y_{2} \varepsilon, 0,1\right)$,

$$
A \cdot Y=((Y V \wedge 1 S) O \wedge A S) V \wedge O U=\left[1,0, a_{1} y_{2} \varepsilon,\right] \wedge[0,1,0]=\left(a_{1} y_{2} \varepsilon, 0,1\right)
$$

and

$$
Y \cdot A=((A V \wedge 1 S) O \wedge Y S) V \wedge O U=\left[1,0,\left(y_{2} \varepsilon\right)\left(a_{1}+a_{2} \varepsilon\right)\right] \wedge[0,1,0]=\left(y_{2} a_{1} \varepsilon, 0,1\right)
$$

we have $A \cdot Y \sim Y$ and $Y \cdot A \sim Y$. 
Competing interests

The authors declare that they have no competing interests.

\section{Authors' contributions}

Both authors have contributed equally to this paper. Both authors read and approved the final manuscript.

\section{Acknowledgements}

Dedicated to Professor Hari M Srivastava.

Received: 11 January 2013 Accepted: 23 April 2013 Published: 7 May 2013

\section{References}

1. Hughes, DR, Piper, FC: Projective Planes. Springer, New York (1973)

2. Baker, CA, Lane, ND, Lorimer, JW: A coordinatization for Moufang-Klingenberg planes. Simon Stevin 65, 3-22 (1991)

3. Keppens, D: Coordinatization of projective Klingenberg planes. Simon Stevin 62, 63-90 (1988)

4. Dayioglu, A, Celik, B: Projective-Klingenberg planes constructed with dual local rings. AIP Conf. Proc. 1389, 308-311 (2011)

5. Conway, JH, Smith, DA: On Quaternions and Octonions. AK Peters, Wellesley (2003)

doi:10.1186/1029-242X-2013-230

Cite this article as: Çelik and Erdoğan: On addition and multiplication of points in a certain class of projective Klingenberg planes. Journal of Inequalities and Applications 2013 2013:230.

\section{Submit your manuscript to a SpringerOpen ${ }^{\circ}$ journal and benefit from:}

- Convenient online submission

- Rigorous peer review

- Immediate publication on acceptance

- Open access: articles freely available online

- High visibility within the field

- Retaining the copyright to your article 